S2 table. Description of the canonical pathways containing the DEGs in CCC myocardium.

\begin{tabular}{|c|c|c|c|c|}
\hline Canonical Pathways & p-value & Ratio & z-score & Molecules \\
\hline Th1 and Th2 Activation Pathway & $2,5 \mathrm{E}-20$ & $2,69 \mathrm{E}-01$ & & $\begin{array}{l}\text { CCR5, CD247, CD3D, CD3E, CD3G, CD40LG, CD8A, CXCR3, } \\
\text { CXCR4, CXCR6, GFI1, HLA-A, HLA-DMA, HLA-DMB, HLA-DOA, } \\
\text { HLA-DOB, HLA-DPA1, HLA-DPB1, HLA-DQB1, HLA-DQB2, HLA- } \\
\text { DRB1, HLA-DRB5, ICOS, IFNG, IKZF1, IL10RA, IL12RB1, } \\
\text { IL12RB2, IL17RB, IL18, IL2RB, IL2RG, IL4, ITGB2, JAK3, KLRC1, } \\
\text { KLRD1, NFATC2, NOTCH2, PIK3CG, PIK3R1, PIK3R5, PRKCQ, } \\
\text { RUNX3, SOCS1, STAT1, STAT4, TBX21, TGFBR2, VAV1 }\end{array}$ \\
\hline Th1 Pathway & $1,0 \mathrm{E}-19$ & 3,09E-01 & 5,209 & $\begin{array}{l}\text { CCR5, CD247, CD3D, CD3E, CD3G, CD40LG, CD8A, CXCR3, } \\
\text { HLA-A, HLA-DMA, HLA-DMB, HLA-DOA, HLA-DOB, HLA-DPA1, } \\
\text { HLA-DPB1, HLA-DQB1, HLA-DQB2, HLA-DRB1, HLA-DRB5, } \\
\text { ICOS, IFNG, IL10RA, IL12RB1, IL12RB2, IL18, IL4, ITGB2, JAK3, } \\
\text { KLRC1, KLRD1, NFATC2, NOTCH2, PIK3CG, PIK3R1, PIK3R5, } \\
\text { PRKCQ, RUNX3, SOCS1, STAT1, STAT4, TBX21, VAV1 }\end{array}$ \\
\hline Th2 Pathway & $4,0 \mathrm{E}-17$ & 2,73E-01 & 1,800 & $\begin{array}{l}\text { CCR5, CD247, CD3D, CD3E, CD3G, CXCR4, CXCR6, GFI1, HLA- } \\
\text { A, HLA-DMA, HLA-DMB, HLA-DOA, HLA-DOB, HLA-DPA1, HLA- } \\
\text { DPB1, HLA-DQB1, HLA-DQB2, HLA-DRB1, HLA-DRB5, ICOS, } \\
\text { IFNG, IKZF1, IL12RB1, IL12RB2, IL17RB, IL2RB, IL2RG, IL4, } \\
\text { ITGB2, JAK3, NFATC2, NOTCH2, PIK3CG, PIK3R1, PIK3R5, } \\
\text { PRKCQ, RUNX3, STAT4, TBX21, TGFBR2, VAV1 }\end{array}$ \\
\hline iCOS-iCOSL Signaling in T Helper Cells & $2,0 \mathrm{E}-13$ & $2,6 \mathrm{E}-01$ & 5,014 & $\begin{array}{l}\text { CAMK4, CD247, CD3D, CD3E, CD3G, CD40LG, GRAP2, HLA-A, } \\
\text { HLA-DMA, HLA-DMB, HLA-DOA, HLA-DOB, HLA-DQB1, HLA- } \\
\text { DRB1, HLA-DRB5, ICOS, IKBKE, IL2RB, IL2RG, INPPDD, ITK, LAT, } \\
\text { LCK, LCP2, NFATC2, PIK3CG, PIK3R1, PIK3R5, PRKCQ, PTPRC, } \\
\text { TRAT1, VAV1, ZAP70 }\end{array}$ \\
\hline CD28 Signaling in T Helper Cells & $1,6 \mathrm{E}-12$ & 2,43E-01 & 3,273 & $\begin{array}{l}\text { CAMK4, CARD11, CD247, CD3D, CD3E, CD3G, CDC42, CTLA4, } \\
\text { GRAP2, HLA-A, HLA-DMA, HLA-DMB, HLA-DOA, HLA-DOB, HLA- } \\
\text { DQB1, HLA-DRB1, HLA-DRB5, IKBKE, ITK, LAT, LCK, LCP2, } \\
\text { NFATC2, PIK3CG, PIK3R1, PIK3R5, PRKCQ, PTPN6, PTPRC, } \\
\text { SYK, VAV1, WAS, ZAP70 }\end{array}$ \\
\hline Natural Killer Cell Signaling & $6,3 \mathrm{E}-12$ & 2,44E-01 & & $\begin{array}{l}\text { CD244, CD247, CD300A, FCGR3A/FCGR3B, HCST, INPP5D, } \\
\text { KIR2DS2 (includes others), KLRB1, KLRC1, KLRC3, KLRC4- } \\
\text { KLRK1/KLRK1, KLRD1, LAIR1, LAT, LCK, LCP2, LILRB1, NCR3, }\end{array}$ \\
\hline
\end{tabular}



Immune Cells Arthritis

\section{B Cell Development}

3,6E-09

Primary Immunodeficiency Signaling

Autoimmune Thyroid Disease Signaling

$6,5 \mathrm{E}-09$

3,33E-01

8,9E-09

3,27E-01

9,3E-09 2,32E-01
PIK3CG, PIK3R1, PIK3R5, PRKCB, PRKCQ, PTPN6, RAC2, SH2D1A, SYK, TYROBP, VAV1, VAV3, ZAP70

BTK, CAMK4, CARD11, CD247, CD3D, CD3E, CD3G, CD8A CD8B, CTLA4, GRAP2, IKBKE, ITK, LAT, LCK, LCP2, NFATC2, PIK3CG, PIK3R1, PIK3R5, PRKCQ, PTPN7, PTPRC, PTPRH RASGRP1, VAV1, VAV3, ZAP70

CD74, HLA-A, HLA-DMA, HLA-DMB, HLA-DOA, HLA-DOB, HLADPA1, HLA-DPB1, HLA-DQB1, HLA-DQB2, HLA-DRB1, HLADRB5, HLA-E, HLA-F, IFNG, MR1

CD40LG, HLA-A, HLA-DMA, HLA-DMB, HLA-DOA, HLA-DOB, HLA-DQB1, HLA-DRB1, HLA-DRB5, ICOS, IFNG, IL10RA, IL12RB1, IL12RB2, IL18, IL21R, IL2RG, IL4, STAT1, STAT4, TBX21, TGFBR2

CCL3, CCL3L3, CCL4, CCL5, CCR7, CD40LG, CD8A, CD8B CXCL10, HLA-A, HLA-DRB1, HLA-DRB5, HLA-E, HLA-F, IFNG, IL18, IL4, TLR3, TLR5, TLR6, TLR8, TNFRSF17, TNFSF13, TNFSF13B

CCR7, CD226, CD40LG, CD69, FASLG, HLA-A, HLA-DRB1, HLA DRB5, HLA-E, HLA-F, IFNG, IL15RA, IL18, IL2RB, IL2RG, IL4, ITGAL, KLRC4-KLRK1/KLRK1, KLRD1, LTB, NCR3, PRF1, TLR3, TYROBP

CD40LG, CD79A, FASLG, HLA-A, HLA-DMA, HLA-DMB, HLADOA, HLA-DOB, HLA-DQB1, HLA-DRB1, HLA-DRB5, IFNG, IL18, IL23A, IL4, LTB, SLAMF1, TLR3, TLR5, TLR6, TLR8, TNFRSF17, TNFSF13, TNFSF13B

CCL3, CCL4, CCL5, CCR5, CXCL10, CXCL11, CXCL9, CXCR3

ATP2A2, ATP2A3, CAMK4, CD247, CD3D, CD3E, CD3G, HLA-A HLA-DMA, HLA-DMB, HLA-DOA, HLA-DOB, HLA-DQB1, HLADRB1, HLA-DRB5, LCK, NFATC2, PRKCB, PRKCQ, ZAP70

CD19, CD79A, HLA-A, HLA-DMA, HLA-DMB, HLA-DOA, HLA-DOB, HLA-DQB1, HLA-DRB1, HLA-DRB5, IL7, IL7R, PTPRC, SPN

BLNK, BTK, CD19, CD3D, CD3E, CD40LG, CD79A, CD8A, ICOS, IGLL1/IGLL5, IL2RG, IL7R, JAK3, LCK, PTPRC, ZAP70

CD40LG, CGA, FASLG, GZMB, HLA-A, HLA-DMA, HLA-DMB, HLADOA, HLA-DOB, HLA-DQB1, HLA-DRB1, HLA-DRB5, HLA-E, HLAF, IL4, PRF1

AP1S3, CD247, CD3D, CD3E, CD3G, CD8A, CD8B, CTLA4 GRAP2, HLA-A, LAT, LCK, LCP2, PIK3CG, PIK3R1, PIK3R5, PPM1J, PPP2R2B, PTPN22, PTPN6, SYK, TRAT1, ZAP70 
Macrophages and Monocytes

\section{$9,5 \mathrm{E}-07$}

2,02E-01

Complement System

9,8E-07

3,16E-01

1,0E-06

2,09E-01

Dendritic Cell Maturation
2,2E-06 1,52E-01
CARD11, CD247, CD3D, CD3E, CD3G, GRAP2, HLA-A, HLA-DMA HLA-DMB, HLA-DOA, HLA-DOB, HLA-DQB1, HLA-DRB1, HLADRB5, IKBKE, LAT, LCK, LCP2, NFATC2, PIK3CG, PIK3R1, PIK3R5, PRKCQ, RAC2, VAV1, VAV3, ZAP70

ARHGAP4, ARHGAP9, BCAR1, BTK, CDC42, CLDN14, CLDN3, CXCR4, CYBA, ITGA4, ITGAL, ITGB2, ITK, MMP25, MMP9, NCF1, NCF2, NCF4, PIK3CG, PIK3R1, PIK3R5, PRKCB, PRKCQ, PTK2, PTK2B, RAC2, RASGRP1, RHOH, SELPLG, SPN, THY1, VAV1, VAV3, WAS, WIPF1

FASLG, GZMB, HLA-A, HLA-DMA, HLA-DMB, HLA-DOA, HLADOB, HLA-DQB1, HLA-DRB1, HLA-DRB5, HLA-E, HLA-F, IFNG IL18, PRF1

CASP8, CD247, CD3D, CD3E, CD3G, CYCS, FASLG, GZMB, HLAA, HLA-DMA, HLA-DMB, HLA-DOA, HLA-DOB, HLA-DQB1, HLADRB1, HLA-DRB5, HLA-E, HLA-F, IFNG, IKBKE, PRF1, SOCS1, STAT1

C5, CCL14, CCL17, CCL19, CCL28, CCL3, CCL3L3, CCL4, CCL5, CLDN14, CLDN3, CXCL10, CXCL11, CXCL16, CXCL9, CXCR2, CXCR4, HRH2, IL18, IL18RAP, ITGA4, ITGAL, ITGB2, MMP25, MMP9, SDC1, SELL, SELPLG, THY1, XCL1

BLNK, BTK, CAMK4, CD247, CD3D, CD3E, CD3G, CD79A, FCGR3A/FCGR3B, GATA4, HLA-A, HLA-DMA, HLA-DMB, HLADOA, HLA-DOB, HLA-DQB1, HLA-DRB1, HLA-DRB5, IKBKE, ITK, LAT, LCK, LCP2, NFATC2, PIK3CG, PIK3R1, PIK3R5, PLCB2, PRKCQ, SYK, ZAP70

CD40LG, FASLG, GZMB, HLA-A, HLA-DMA, HLA-DMB, HLA-DOA HLA-DOB, HLA-DPA1, HLA-DPB1, HLA-DQB1, HLA-DQB2, HLADRB1, HLA-DRB5, HLA-E, HLA-F, IFNG, IL4, PRF1

CDC42, FCGR3A/FCGR3B, FGR, FYB1, INPP5D, LCP2, MYO5A, NCF1, NCK2, PIK3CG, PIK3R1, PLD4, PRKCB, PRKCQ, PTK2B, RAC2, SYK, VAV1, VAV3, WAS

1,414 C1QA, C1QB, C1QC, C1S, C2, C5, C8G, CD59, CFB, CFH, ITGAX ITGB2

HLA-A, HLA-DMA, HLA-DMB, HLA-DOA, HLA-DOB, HLA-DQB1, HLA-DRB1, HLA-DRB5, IL2RG, IL4, INPP5D, IRF4, JAK3, NFATC2, PIK3CG, PIK3R1, PIK3R5, PTPN6, SOCS1

CCR7, CD1B, CD1C, CD40LG, COL1A2, COL3A1, FCGR3A/FCGR3B, HLA-A, HLA-DMA, HLA-DMB, HLA-DOA, HLADOB, HLA-DQB1, HLA-DRB1, HLA-DRB5, IKBKE, IL18, IL23A 
Role of JAK1 and JAK3 in yc Cytokine Signaling

$2,5 \mathrm{E}-06$

$2,25 \mathrm{E}-0$

OX40 Signaling Pathway

Nur77 Signaling in T Lymphocytes

Cytotoxic T Lymphocyte-mediated Apoptosis of Target Cells

Agranulocyte Adhesion and Diapedesis

Cdc42 Signaling

Role of Pattern Recognition Receptors in

Recognition of Bacteria and Viruses

Atherosclerosis Signaling

Phagosome Formation

Hepatic Fibrosis / Hepatic Stellate Cell Activation

Systemic Lupus Erythematosus Signaling
9,5E-05 1,34E-01

9,5E-05 1,34E-01

3,2E-06 2,02E-01

$-1,342$

6,9E-06 2,33E-01

8,9E-06 3,12E-0

1,7E-05

1,42E-01

1,7E-05 1,48E-01

0,000

4,7E-05 1,53E-01

4,000

5,2E-05 1,56E-01

8,5E-05 1,56E-01

1,8E-04 1,22E-01
IRF8, LTB, LY75, PIK3CG, PIK3R1, PIK3R5, PLCB2, STAT1, STAT4, TLR3, TYROBP

BLNK, IL15RA, IL21R, IL2RB, IL2RG, IL4, IL7, IL7R, JAK3, PIK3CG, PIK3R1, PIK3R5, PTK2B, SOCS1, STAT1, SYK

CD247, CD3D, CD3E, CD3G, HLA-A, HLA-DMA, HLA-DMB, HLADOA, HLA-DOB, HLA-DPA1, HLA-DPB1, HLA-DQB1, HLA-DQB2, HLA-DRB1, HLA-DRB5, HLA-E, HLA-F, TRAF5

CAMK4, CD247, CD3D, CD3E, CD3G, CYCS, HLA-A, HLA-DMA HLA-DMB, HLA-DOA, HLA-DOB, HLA-DQB1, HLA-DRB1, HLADRB5

-0,333 CASP8, CD247, CD3D, CD3E, CD3G, CYCS, FASLG, GZMB, HLAA, PRF1

C5, CCL14, CCL17, CCL19, CCL28, CCL3, CCL3L3, CCL4, CCL5, CLDN14, CLDN3, CXCL10, CXCL11, CXCL16, CXCL9, CXCR2, CXCR4, IL18, ITGA4, ITGB2, ITGB7, MMP25, MMP9, MYH6, SELL, SELPLG, XCL1

CD247, CD3D, CD3E, CD3G, CDC42, FGD3, HLA-A, HLA-DMA HLA-DMB, HLA-DOA, HLA-DOB, HLA-DPA1, HLA-DPB1, HLADQB1, HLA-DQB2, HLA-DRB1, HLA-DRB5, HLA-E, HLA-F, ITGA4, ITK, MPRIP, VAV1, WAS, WIPF1

C1QA, C1QB, C1QC, C5, CCL5, IFIH1, IFNG, IL18, IL4, NLRC4, NLRP3, PIK3CG, PIK3R1, PIK3R5, PRKCB, PRKCQ, SYK, TLR3, TLR5, TLR6, TLR8

ALOX15B, APOA1, CCR2, CD40LG, CMA1, COL1A2, COL3A1, CXCR4, IFNG, IL18, ITGA4, ITGB2, MMP9, PLA2G4F, PLA2G7, PLAAT4, PLB1, SELPLG, SERPINA1, TNFSF14

FCAR, FCGR3A/FCGR3B, FNBP1, INPP5D, ITGA4, MRC1, PIK3CG, PIK3R1, PIK3R5, PLCB2, PRKCB, PRKCQ, RHOD, $\mathrm{RHOH}$, SYK, TLR3, TLR5, TLR6, TLR8

CCL5, CCR5, CCR7, CD40LG, COL16A1, COL1A2, COL3A1, COL4A3, COL4A4, COL5A1, COL5A2, COL6A6, CXCL9, CXCR3, FASLG, IFNG, IGF1, IL10RA, IL18RAP, IL4, MET, MMP9, MYH6, STAT1, TGFBR2

C5, C8G, CAMK4, CD247, CD3D, CD3E, CD3G, CD40LG, CD72, CD79A, FCGR3A/FCGR3B, HLA-A, HLA-E, HLA-F, IL18, INPP5D, KNG1, LAT, LCK, NFATC2, PIK3CG, PIK3R1, PIK3R5, PIM2, PTPN6, PTPRC, RNU2-2P, TNFSF13B 
Tumoricidal Function of Hepatic Natural Killer Cells

Production of Nitric Oxide and Reactive Oxygen

Species in Macrophages

Regulation of IL-2 Expression in Activated and Anergic T Lymphocytes

Role of Macrophages, Fibroblasts and Endothelial

Cells in Rheumatoid Arthritis
1,07E-01
BLK, BTK, FASLG, FGR, FNBP1, ITGA4, ITK, JAK3, LCK, PIK3CG PIK3R1, PIK3R5, PRKCB, PRKCQ, PTK2, PTK2B, RHOD, RHOH, STAT1, STAT4, VAV1, VAV3, WAS

BLK, BLNK, BTK, CAMK4, CD19, CD79A, DAPP1, IKBKE, IL4, INPP5D, NFATC2, PIK3CG, PIK3R1, PLCB2, PRKCB, PTPRC, SYK, VAV1, VAV3

ADCY7, ARHGEF16, BLNK, BTK, CAMK4, CD247, CD3D, CD3E, CD3G, CD79A, FNBP1, GRAP2, HDAC10, ITGA4, ITK, LAT, LCK, LCP2, MPRIP, NFATC2, PLA2G4F, PLCB2, PLD4, PRKCB, PRKCQ, RHOD, RHOH, SYK, ZAP70

APBB1IP, BLNK, BTK, CAMK4, CD19, CD79A, CDC42, DAPP1, IKBKE, INPP5D, NFATC2, PIK3CG, PIK3R1, PIK3R5, PRKCB, PRKCQ, PTK2, PTK2B, PTPN6, PTPRC, RAC2, SYK, VAV1, VAV3 CAMK4, CCL3, CCL4, CCL5, CCR5, CD247, CD3D, CD3E, CD3G, FASLG, PRKCB, PRKCQ, PTK2B

CASP8, CYCS, ENDOG, FASLG, GZMB, ITGAL, PRF1

APOA1, CYBA, FNBP1, IFNG, IKBKE, IL4, IRF8, JAK3, NCF1, NCF2, NCF4, PIK3CG, PIK3R1, PIK3R5, PPM1J, PPP1CC PPP2R2B, PRKCB, PRKCQ, PTPN6, RHOD, RHOH, SERPINA1, STAT1

CAMK4, CARD11, CD247, CD3D, CD3E, CD3G, IKBKE, LAT, NFATC2, TGFBR2, VAV1, VAV3, ZAP70

C5, CAMK4, CCL5, CEBPE, FCGR3A/FCGR3B, FRZB, IKBKE, IL16, IL18, IL18RAP, IL7, IRAK3, LEF1, LTB, NFATC2, PIK3CG, PIK3R1, PIK3R5, PLCB2, PRKCB, PRKCQ, SFRP4, SOCS1, TLR3, TLR5, TLR6, TLR8, TNFSF13B, TRAF1, TRAF5, WNT10A, WNT3, WNT3A

ADCY7, ADORA3, ADRB1, CAMK4, CXCR2, DRD4, GRM1, HRH2, IKBKE, P2RY12, P2RY13, P2RY14, PDE4A, PDE4D, PDE6A, PDE9A, PIK3CG, PIK3R1, PIK3R5, PLCB2, PRKCB, PTGDR, PTGER3, PTH1R, PTK2B, RASGRP1, RGS10, RGS18, SYNGAP1, VIPR2

APOA1, CD40LG, IFNG, IKBKE, IL12RB1, IL12RB2, IL18, IL23A IL4, IRF8, JMJD6, PIK3CG, PIK3R1, PIK3R5, PRKCB, PRKCQ, SERPINA1, STAT1, STAT4

ACP5, BMP6, BMP7, CAMK4, CSF1R, CTSK, FRZB, IFNG, IGF1, IKBKE, IL18, IL18RAP, IL4, IL7, LEF1, NFATC2, PIK3CG, PIK3R1, 
IL-15 Signaling

Inflammasome pathway

Granzyme B Signaling

NF-kB Signaling

Fc Epsilon RI Signaling

Sperm Motility

Axonal Guidance Signaling

Chemokine Signaling

TREM1 Signaling

IL-2 Signaling

Hematopoiesis from Pluripotent Stem Cells

Molecular Mechanisms of Cancer

\section{Paxillin Signaling \\ p70S6K Signaling}

3-phosphoinositide Biosynthesis
4,1E-03

$9,01 \mathrm{E}-02$

4,1E-03 1,47E-

4,5E-03 1,45E-01

2,714

1,6E-03 1,56E-01

2,61E-01

$3,12 \mathrm{E}-01$

$1,17 \mathrm{E}-0$

1,28E-01

1,21E-01

1,069

4,8E-03 1,52E-01

5,5E-03 1,7E-01

5,8E-03 9,16E-02

$\begin{array}{lll}6,2 \mathrm{E}-03 & 1,24 \mathrm{E}-01 & 1,000 \\ 6,3 \mathrm{E}-03 & 1,17 \mathrm{E}-01 & 3,000 \\ 6,5 \mathrm{E}-03 & 1,04 \mathrm{E}-01 & \end{array}$

PIK3R5, PTK2B, RUNX2, SFRP4, TRAF5, WNT10A, WNT3, WNT3A

FASLG, IL15RA, IL2RB, IL2RG, IL4, JAK3, LCK, PIK3CG, PIK3R1, PIK3R5, PTK2, SYK

AIM2, CASP8, IL18, NLRC4, NLRP3, PYCARD

CASP8, CYCS, ENDOG, GZMB, PRF1

CARD11, CASP8, CD40LG, IL18, IRAK3, LCK, PIK3CG, PIK3R1, PIK3R5, PRKCB, PRKCQ, TGFBR2, TLR3, TLR5, TLR6, TLR8 TNFAIP3, TNFRSF17, TNFSF13B, TRAF5, ZAP70

BTK, GRAP2, IL4, INPP5D, LAT, LCP2, PIK3CG, PIK3R1, PIK3R5, PLA2G4F, PRKCB, PRKCQ, RAC2, SYK, VAV1, VAV3

ATP1A4, CAMK4, CATSPER1, CATSPER2, NPPA, PDE4A, PDE4D, PLA2G4F, PLA2G7, PLAAT4, PLB1, PLCB2, PRKCB, PRKCQ, PTK2, PTK2B, PTK6

ABLIM2, ADAM11, ADAM28, ADAM8, AOPEP, BCAR1, BMP6, BMP7, CDC42, CXCR4, GLIS1, GLIS2, IGF1, ITGA4, LRRC4C, MET, MICAL1, MMP9, NCK2, NFATC2, PIK3CG, PIK3R1, PIK3R5, PLCB2, PLXNC1, PRKCB, PRKCQ, PTK2, RAC2, RHOD, SEMA4D, SEMA6B, SLIT2, SRGAP2, TUBA3C/TUBA3D, TUBB2A, WAS, WIPF1, WNT10A, WNT3, WNT3A

CAMK4, CCL4, CCL5, CCR5, CXCR4, MPRIP, PIK3CG, PLCB2, PRKCB, PTK2, PTK2B

CCL3, IL18, ITGAX, NLRC3, NLRC4, NLRP3, TLR3, TLR5, TLR6, TLR8, TYROBP

2,530 IL2RB, IL2RG, JAK3, LCK, PIK3CG, PIK3R1, PIK3R5, PTK2B SOCS1, SYK

CD247, CD3D, CD3E, CD3G, CD8A, CD8B, IL4, IL7

ADCY7, ARHGEF16, AURKA, BCL2L11, BMP6, BMP7, CASP8, CDC42, CDKN2A, CHEK2, CYCS, E2F2, FANCD2, FASLG FNBP1, HHAT, ITGA4, JAK3, LEF1, PIK3CG, PIK3R1, PIK3R5, PLCB2, PRKCB, PRKCQ, PTK2, RAC2, RASGRP1, RHOD, $\mathrm{RHOH}$ SYNGAP1, TGFBR2, WNT10A, WNT3, WNT3A

BCAR1, CDC42, ITGA4, ITGAD, ITGAL, ITGAX, ITGB2, ITGB7, NCK2, PIK3CG, PIK3R1, PIK3R5, PTK2, PTK2B

BTK, CD19, CD79A, F2R, F2RL2, IL2RG, IL4, PIK3CG, PIK3R1, PIK3R5, PLCB2, PPM1J, PPP2R2B, PRKCB, PRKCQ, SYK

ACP5, CD19, CILP, DUSP13, ICOS, LCK, MET, NUDT4, PIK3CG, PIK3R1, PIK3R5, PIP4K2A, PPP1CC, PPP1R1A, PTPN22, PTPN6, PTPN7, PTPRC, PTPRH, PTPRO, TRAT1, VAV1 
IL-8 Signaling

Sphingosine-1-phosphate Signaling

Macropinocytosis Signaling

Role of PKR in Interferon Induction and Antivira Response

Superpathway of Inositol Phosphate Compounds

Ga12/13 Signaling

Eicosanoid Signaling

Role of JAK1, JAK2 and TYK2 in Interferon Signaling

Role of Hypercytokinemia/hyperchemokinemia in

the Pathogenesis of Influenza

NF-kB Activation by Viruses

Actin Nucleation by ARP-WASP Complex Neuroprotective Role of THOP1 in Alzheimer's Disease

UVA-Induced MAPK Signaling

FcyRIIB Signaling in B Lymphocytes

Reelin Signaling in Neurons

Lymphotoxin $\beta$ Receptor Signaling

L-15 Production

Integrin Signaling

Gaq Signaling
7,2E-03 1,04E-01

3,710

7,2E-03 1,18E-0

1,069

7,2E-03 1,36E-01

1,414

7,8E-03 1,75E-01

8,7E-03 9,73E-02

9,5E-03 1,15E-01

2,840

1,0E-02 1,29E-01

1,633

1,1E-02 2,08E-01

0,000

1,1E-02 1,63E-01

1,2E-02 1,26E-01

3,162

1,5E-02 1,43E-01

1,7E-02 1,52E-01

1,7E-02 1,17E-01

1,7E-02 1,4E-01

1,8E-02 1,2E-01

1,9E-02 1,3E-01

1,9E-02 1,85E-01

1,9E-02 9,55E-02

1,9E-02 1,01E-01
ANGPT1, CXCR2, FNBP1, IKBKE, IRAK3, ITGAX, ITGB2, MMP9, NCF2, PIK3CG, PIK3R1, PIK3R5, PLCB2, PLD4, PRKCB, PRKCQ, PTK2, PTK2B, RAC2, RHOD, RHOH

ADCY7, CASP8, FNBP1, NAAA, PIK3CG, PIK3R1, PIK3R5, PLCB2, PTK2, PTK2B, RHOD, RHOH, S1PR4, SMPD3, SPHK1

CDC42, CSF1R, ITGB2, ITGB7, MET, MRC1, PIK3CG, PIK3R1, PIK3R5, PRKCB, PRKCQ

CASP8, CYCS, IFNG, IKBKE, STAT1, TLR3, TRAF5

ACP5, CD19, CILP, DUSP13, ICOS, INPP5D, LCK, MET, NUDT4, PIK3CG, PIK3R1, PIK3R5, PIP4K2A, PLCB2, PLD4, PPP1CC, PPP1R1A, PTPN22, PTPN6, PTPN7, PTPRC, PTPRH, PTPRO, TRAT1, VAV1

BTK, CDC42, CDH16, CDH9, F2R, F2RL2, IKBKE, LPAR2, PIK3CG, PIK3R1, PIK3R5, PTK2, PTK2B, VAV1, VAV3

ALOX15B, ALOX5AP, CYSLTR1, DPEP2, LTC4S, PLA2G4F PLA2G7, PLAAT4, PLB1, PTGDR, PTGER3

CGA, IFNG, PTPN6, SOCS1, STAT1

CCL3, CCL4, CCL5, CCR5, CXCL10, IFNG, IL18

CCR5, IKBKE, ITGA4, ITGAL, ITGB2, LCK, PIK3CG, PIK3R1, PIK3R5, PRKCB, PRKCQ

0,816 CDC42, FNBP1, ITGA4, NCK2, RHOD, RHOH, WAS, WIPF1

HLA-A, HLA-E, HLA-F, IFNG, KNG1, MMP9, PNOC

2,714 CYCS, PARP10, PARP15, PARP4, PARP8, PIK3CG, PIK3R1, PIK3R5, PLCB2, SMPD3, STAT1, ZC3HAV1

1,414 BLNK, BTK, CD79A, INPP5D, PIK3CG, PIK3R1, PIK3R5, SYK

ARHGEF16, BLK, FGR, ITGA4, ITGAL, ITGB2, LCK, MAP4K1, PIK3CG, PIK3R1, PIK3R5

2,333 CYCS, IKBKE, LTB, PIK3CG, PIK3R1, PIK3R5, TNFSF14, TRAF1, TRAF5

JAK3, PTK2, PTK2B, PTK6, STAT1

2,524 BCAR1, CDC42, FNBP1, ITGA4, ITGAD, ITGAL, ITGAX, ITGB2, ITGB7, MPRIP, NCK2, PIK3CG, PIK3R1, PIK3R5, PTK2, RAC2, RHOD, RHOH, TSPAN5, WAS, WIPF1

BTK, CAMK4, FNBP1, GRM1, IKBKE, NFATC2, PIK3CG, PIK3R1, PIK3R5, PLCB2, PLD4, PRKCB, PRKCQ, PTK2B, RGS18, RHOD, $\mathrm{RHOH}$ 
Acute Myeloid Leukemia Signaling

April Mediated Signaling

Glioma Invasiveness Signaling

ILK Signaling

Virus Entry via Endocytic Pathways

PTEN Signaling

cAMP-mediated signaling

Caveolar-mediated Endocytosis Signaling

Ephrin A Signaling

B Cell Activating Factor Signaling

Toll-like Receptor Signaling

Retinoic acid Mediated Apoptosis Signaling

CD40 Signaling

Colorectal Cancer Metastasis Signaling

Death Receptor Signaling

Differential Regulation of Cytokine Production in Intestinal Epithelial Cells by IL-17A and IL-17F

Regulation of Actin-based Motility by Rho

IL-9 Signaling

Pancreatic Adenocarcinoma Signaling

Myc Mediated Apoptosis Signaling

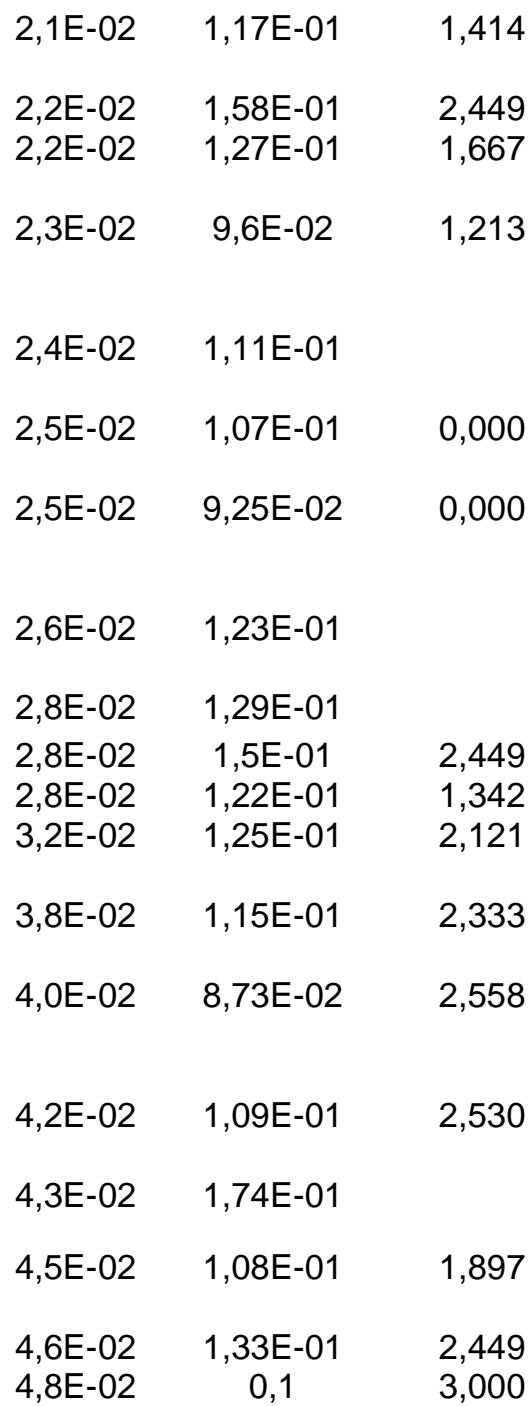

5,1E-02 1,14E-01
CSF1R, CSF2RA, FLT3, FLT3LG, IDH2, LEF1, PIK3CG, PIK3R1, PIK3R5, PIM1, PIM2

IKBKE, NFATC2, TNFRSF17, TNFSF13, TRAF1, TRAF5

F2R, FNBP1, MMP9, PIK3CG, PIK3R1, PIK3R5, PTK2, RHOD, $\mathrm{RHOH}$

CDC42, FBLIM1, FERMT2, FNBP1, ITGB2, ITGB7, LEF1, MMP9, MYH6, NCK2, PIK3CG, PIK3R1, PIK3R5, PPM1J, PPP2R2B, PTK2, RHOD, RHOH, TMSB10/TMSB4X

CDC42, HLA-A, ITGA4, ITGAL, ITGB2, ITGB7, PIK3CG, PIK3R1, PIK3R5, PRKCB, PRKCQ, RAC2

BCAR1, BCL2L11, CDC42, FASLG, IKBKE, INPP5D, ITGA4 PIK3CG, PIK3R1, PIK3R5, PTK2, RAC2, TGFBR2

ADCY7, ADORA3, ADRB1, AKAP10, CAMK4, CXCR2, DRD4, HRH2, P2RY12, P2RY13, P2RY14, PDE4A, PDE4D, PDE6A, PDE9A, PTGDR, PTGER3, PTH1R, RGS10, RGS18, VIPR2

CD48, DYRK3, HLA-A, ITGA4, ITGAD, ITGAL, ITGAX, ITGB2, ITGB7

BCAR1, CDC42, PIK3CG, PIK3R1, PIK3R5, PTK2, VAV1, VAV3

IKBKE, NFATC2, TNFRSF17, TNFSF13B, TRAF1, TRAF5

IL18, IRAK3, TLR3, TLR5, TLR6, TLR8, TNFAIP3, TRAF1, UBD

CASP8, CYCS, IFNG, PARP10, PARP15, PARP4, PARP8, ZC3HAV1

CD40LG, IKBKE, JAK3, PIK3CG, PIK3R1, PIK3R5, TNFAIP3, TRAF1, TRAF5

ADCY7, FNBP1, IFNG, JAK3, LEF1, MMP25, MMP9, PIK3CG,

PIK3R1, PIK3R5, PTGER3, RHOD, RHOH, STAT1, TGFBR2, TLR3, TLR5, TLR6, TLR8, WNT10A, WNT3, WNT3A

ARHGDIB, CASP8, CYCS, FASLG, IKBKE, PARP10, PARP15, PARP4, PARP8, ZC3HAV1

CCL3, CCL4, CCL5, IFNG

CDC42, FNBP1, ITGA4, MPRIP, PIP4K2A, RAC2, RHOD, RHOH, WAS, WIPF 1

IL2RG, JAK3, PIK3CG, PIK3R1, PIK3R5, STAT1

BRCA2, CDC42, CDKN2A, E2F2, JAK3, MMP9, PIK3CG, PIK3R1,

PIK3R5, PLD4, STAT1, TGFBR2

CASP8, CDKN2A, CYCS, FASLG, IGF1, PIK3CG, PIK3R1, PIK3R5 
Actin Cytoskeleton Signaling

iNOS Signaling

Glioblastoma Multiforme Signaling

Growth Hormone Signaling

Basal Cell Carcinoma Signaling

D-myo-inositol-5-phosphate Metabolism

fMLP Signaling in Neutrophils

HER-2 Signaling in Breast Cancer

Protein Kinase A Signaling

Acute Phase Response Signaling

Cardiac $\beta$-adrenergic Signaling

Differential Regulation of Cytokine Production in Macrophages and T Helper Cells by IL-17A and IL-17F

Tryptophan Degradation to 2-amino-3-

carboxymuconate Semialdehyde

V-glutamyl Cycle

Semaphorin Signaling in Neurons

Regulation of the Epithelial-Mesenchymal

Transition Pathway

Factors Promoting Cardiogenesis in Vertebrates

Angiopoietin Signaling
5,4E-02 8,62E-02

5,5E-02 1,28E-01

5,8E-02 9,15E-02

6,0E-02 1,06E-01

6,2E-02 1,1E-01

6,8E-02 8,93E-02

7,1E-02 9,38E-02

7,1E-02 1,02E-0

7,4E-02 7,67E-02

7,6E-02

8,77E-02

1,000

8,1E-02 8,97E-02

8,5E-02 1,67E-0

8,5E-02 1,67E-01

8,7E-02 1,38E-01

8,7E-02 1,13E-01

8,7E-02 8,47E-02

8,9E-02 9,78E-02

8,9E-02 1,01E-01

2,236

1,069

0,000

2,887

0,000
2,357

1,414

$-0,577$

ADCY7, ADRB1, AKAP10, ATP2A2, ATP2A3, PDE4A, PDE4D,

PDE6A, PDE9A, PPM1J, PPP1CC, PPP1R1A, PPP2R2B

CCL3, CCL4, CCL5

HAAO, IDO2, KMO

ANPEP, CHAC2, GGACT, GGTLC1

FNBP1, MET, PTK2, RHOD, RHOH, SEMA4D

CLDN3, JAK3, LEF1, LOX, MET, MMP9, NOTCH2, PARD6B, PIK3CG, PIK3R1, PIK3R5, PYGO1, TGFBR2, WNT10A, WNT3, WNT3A

BMP6, BMP7, GATA4, LEF1, NPPA, PRKCB, PRKCQ, TGFBR2, WNT3

-0,447 ANGPT1, ANGPTL1, DOK2, IKBKE, PIK3CG, PIK3R1, PIK3R5,

BCAR1, CDC42, F2R, FGD3, ITGA4, KNG1, MATK, MPRIP, MYH6, TMSB10/TMSB4X, VAV1, VAV3, WAS

,

BMP6, BMP7, GLIS1, GLIS2, LEF1, WNT10A, WNT3, WNT3A PPP1R1A, PTPN22, PTPN6, PTPN7, PTPRC, PTPRH PTPRO

, NCF1, NCF2, NFATC2, PIK3CG, PIK3R1,

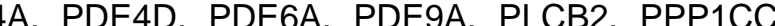
PRKCB, PRKCQ, PTK2, PTK2B, PTPN18, PTPN22, PTPN6, PTPN7, PTPRC, PTPRH, PTPRO, PTPRU, PYGM, RYR1, TGFBR2, TNNI2

PTK2 
Cholecystokinin/Gastrin-mediated Signaling

Unfolded protein response

Intrinsic Prothrombin Activation Pathway

Thrombin Signaling

Cardiomyocyte Differentiation via BMP Receptors

IL-3 Signaling

\section{JAK/Stat Signaling}

Netrin Signaling

Ceramide Signaling

Agrin Interactions at Neuromuscular Junction

FLT3 Signaling in Hematopoietic Progenitor Cells

Wnt/ $\beta$-catenin Signaling

Prolactin Signaling

Renin-Angiotensin Signaling

Small Cell Lung Cancer Signaling

TWEAK Signaling

TGF- $\beta$ Signaling

Glucocorticoid Receptor Signaling

Induction of Apoptosis by HIV1

MSP-RON Signaling Pathway

GM-CSF Signaling

Coagulation System

Role of MAPK Signaling in the Pathogenesis of

Influenza

Ephrin B Signaling

Germ Cell-Sertoli Cell Junction Signaling
9,1E-02 9,43E-02

9,3E-02 1,11E-01

9,5E-02 1,33E-01

1,1E-01 8,13E-02

1,1E-01 1,5E-01

1,1E-01 9,64E-02

1,1E-01 9,64E-02

$1,1 \mathrm{E}-01 \quad 1,14 \mathrm{E}-01$

1,1E-01 9,28E-02

1,1E-01 0,1

1,2E-01 9,41E-02

1,2E-01 8,24E-02

1,3E-01 9,3E-02

1,3E-01

8,53E-02

1,3E-01 9,2E-02

1,3E-01 1,18E-01

1,3E-01 9,2E-02

1,4E-01 7,51E-02

1,4E-01

$1,4 \mathrm{E}-01$

1,4E-01

$1,4 \mathrm{E}-01$

$1,5 \mathrm{E}-01$

$1,5 \mathrm{E}-01$

$1,5 \mathrm{E}-01$

9,33E-02

9,33E-02

7,95E-02
1,265

BCAR1, FNBP1, IL18, PLCB2, PRKCB, PRKCQ, PTK2, PTK2B, $\mathrm{RHOD}, \mathrm{RHOH}$

CEBPE, EDEM1, ERN1, HSPA6, PDIA2, SREBF1

COL1A2, COL3A1, F13A1, KNG1

1,698 ADCY7, ARHGEF16, CAMK4, F2R, F2RL2, FNBP1, GATA4, MPRIP, PIK3CG, PIK3R1, PIK3R5, PLCB2, PRKCB, PRKCQ, PTK2, RHOD, RHOH

BMP7, GATA4, NPPA

1,414 INPP5D, PIK3CG, PIK3R1, PIK3R5, PRKCB, PRKCQ, PTPN6, STAT1

1,414 JAK3, PIK3CG, PIK3R1, PIK3R5, PTPN6, SOCS1, STAT1, STAT4 ABLIM2, NCK2, NFATC2, RAC2, RYR1

-1,414 CYCS, PIK3CG, PIK3R1, PIK3R5, PPM1J, PPP2R2B, S1PR4, SMPD3, SPHK1

0,816 CDC42, ITGA4, ITGAL, ITGB2, PKLR, PTK2, RAC2

2,121 FLT3, FLT3LG, INPP5D, PIK3CG, PIK3R1, PIK3R5, STAT1, STAT4

-0,632 CDKN2A, FRAT1, FRZB, LEF1, MAP4K1, PPM1J, PPP2R2B, SFRP4, SOX10, TGFBR2, UBD, WNT10A, WNT3, WNT3A

2,121 PIK3CG, PIK3R1, PIK3R5, PRKCB, PRKCQ, SOCS1, STAT1, TCF7

2,530 ADCY7, CCL5, PIK3CG, PIK3R1, PIK3R5, PRKCB, PRKCQ, PTK2, PTK2B, PTPN6, STAT1

CYCS, IKBKE, PIK3CG, PIK3R1, PIK3R5, PTK2, TRAF1, TRAF5

0,000

1,342 BMP7, CDC42, MAP4K1, RUNX2, RUNX3, TFE3, TGFBR2, VDR

BAG1, CCL3, CCL5, CD247, CD3D, CD3E, CD3G, ERCC2, FKBP4, HSPA6, IFNG, IKBKE, IL4, JAK3, NFATC2, NPPA, PIK3CG, PIK3R1, PIK3R5, STAT1, TGFBR2, TSC22D3

CASP8, CXCR4, CYCS, FASLG, IKBKE, TRAF1

CCR2, IFNG, ITGB2, PIK3CG, PIK3R1, PIK3R5

CSF2RA, PIK3CG, PIK3R1, PIK3R5, PIM1, PRKCB, STAT1

46E-02 $\quad 1,633$

$1,14 \mathrm{E}-01 \quad 1,000$
F13A1, F2R, KNG1, SERPINA1

CCL5, CXCL10, IFNG, PLA2G4F, PLA2G7, PLAAT4, PLB1

0,816 CDC42, CXCR4, NCK2, PTK2, RAC2, VAV1, VAV3

BCAR1, CDC42, FNBP1, PIK3CG, PIK3R1, PIK3R5, PTK2, RAB8B RAC2, RHOD, RHOH, TGFBR2, TUBA3C/TUBA3D, TUBB2A 


\section{Gustation Pathway}

Neuropathic Pain Signaling In Dorsal Horn Neurons

HGF Signaling

Glioma Signaling

Activation of IRF by Cytosolic Pattern Recognition Receptors

Hematopoiesis from Multipotent Stem Cells

TNFR1 Signaling

Human Embryonic Stem Cell Pluripotency

D-myo-inositol $(1,4,5,6)$-Tetrakisphosphate Biosynthesis

D-myo-inositol $(3,4,5,6)$-tetrakisphosphate

Biosynthesis

3-phosphoinositide Degradation

Breast Cancer Regulation by Stathmin 1

Role of BRCA1 in DNA Damage Response

Role of PI3K/AKT Signaling in the Pathogenesis of

Influenza

Role of NANOG in Mammalian Embryonic Stem

Cell Pluripotency

IL-17A Signaling in Gastric Cells

Leukotriene Biosynthesis

VDR/RXR Activation

GDNF Family Ligand-Receptor Interactions

CD27 Signaling in Lymphocytes

Lipid Antigen Presentation by CD1

Synaptic Long Term Depression

DNA Double-Strand Break Repair by Homologous Recombination

Calcium Transport I
1,5E-01 8,33E-02

1,5E-01 8,47E-02

2,530

1,5E-01 8,47E-02

0,632

1,5E-01 8,47E-02

1,5E-01 9,68E-02

1,5E-01 1,67E-01

$1,6 \mathrm{E}-01 \quad 1,02 \mathrm{E}-01$

1,6E-01 8,11E-02

1,6E-01 8,05E-02

1,6E-01 8,05E-02

1,6E-01 7,93E-02

1,6E-01 7,66E-02

1,7E-01 8,97E-02

1,7E-01 8,97E-02

1,8E-01 8,2E-02

1,8E-01 1,2E-01

1,8E-01 1,2E-01

1,8E-01 8,86E-02

1,8E-01 8,86E-02

1,9E-01 9,62E-02

1,9E-01 1,15E-01

2,0E-01 7,74E-02

2,0E-01 1,43E-01

2,0E-01 1,43E-01

0,000

2,449

2,000

2,309
ADCY7, P2RX3, P2RY12, P2RY13, P2RY14, P2RY2, PDE4A, PDE4D, PDE6A, PDE9A, PLCB2

CAMK4, GRM1, KCNN2, KCNN4, PIK3CG, PIK3R1, PIK3R5, PLCB2, PRKCB, PRKCQ

CDC42, CDKN2A, ITGA4, MET, PIK3CG, PIK3R1, PIK3R5, PRKCB, PRKCQ, PTK2

2,333 CAMK4, CDKN2A, E2F2, IDH2, IGF1, PIK3CG, PIK3R1, PIK3R5 PRKCB, PRKCQ

DHX58, IFIH1, IFIT2, IKBKE, STAT1, ZBP1

IL4, IL7

$0,000 \quad$ CASP8, CDC42, CYCS, IKBKE, TNFAIP3

BMP6, BMP7, LEF1, PIK3CG, PIK3R1, PIK3R5, S1PR4, SPHK1, TGFBR2, WNT10A, WNT3, WNT3A

ACP5, CILP, DUSP13, NUDT4, PPP1CC, PPP1R1A, PTPN22, PTPN6, PTPN7, PTPRC, PTPRH, PTPRO

ACP5, CILP, DUSP13, NUDT4, PPP1CC, PPP1R1A, PTPN22, PTPN6, PTPN7, PTPRC, PTPRH, PTPRO

ACP5, CILP, DUSP13, INPP5D, NUDT4, PPP1CC, PPP1R1A, PTPN22, PTPN6, PTPN7, PTPRC, PTPRH, PTPRO

ADCY7, ARHGEF16, CAMK4, CDC42, E2F2, PIK3CG, PIK3R1, PIK3R5, PLCB2, PPM1J, PPP1CC, PPP2R2B, PRKCB, PRKCQ, TUBA3C/TUBA3D, TUBB2A

BRCA2, CHEK2, E2F2, FANCA, FANCD2, IFNG, STAT1

CCL5, CCR5, IFNG, PIK3CG, PIK3R1, PIK3R5, PLAC8

WNT3, WNT3A

CCL5, CXCL10, CXCL11

DPEP2, GGTLC1, LTC4S

0,378 CCL5, CXCL10, IFNG, PRKCB, PRKCQ, RUNX2, VDR

1,890 CDC42, DOK2, DOK3, GFRA2, PIK3CG, PIK3R1, PIK3R5

1,342 CASP8, CD27, CYCS, IKBKE, TRAF5

CD1B, CD1C, CD3E

GRM1, IGF1, PLA2G4F, PLA2G7, PLAAT4, PLB1, PLCB2, PPM1J, PPP2R2B, PRKCB, PRKCQ, RYR1

BRCA2, LIG1

ATP2A2, ATP2A3 
Sphingomyelin Metabolism

PDGF Signaling

Erythropoietin Signaling

Nitric Oxide Signaling in the Cardiovascular

System

CXCR4 Signaling

ErbB Signaling

LXR/RXR Activation

Thrombopoietin Signaling

Role of CHK Proteins in Cell Cycle Checkpoint

Control

Hepatic Cholestasis

PEDF Signaling

Dopamine-DARPP32 Feedback in cAMP

Signaling

Ovarian Cancer Signaling

LPS-stimulated MAPK Signaling

TNFR2 Signaling

Role of RIG1-like Receptors in Antiviral Innate Immunity

Role of p14/p19ARF in Tumor Suppression

Phospholipases

RANK Signaling in Osteoclasts

HMGB1 Signaling

PAK Signaling

Airway Inflammation in Asthma

Anandamide Degradation

Chronic Myeloid Leukemia Signaling

UDP-N-acetyl-D-glucosamine Biosynthesis II

Apoptosis Signaling
2,0E-01 1,43E-01

$2,0 \mathrm{E}-01$ - $833 \mathrm{E}-0$

2,0E-01 8,54E-02

2,0E-01 7,94E-02

2,1E-01 7,51E-02

2,1E-01 8,16E-02

2,1E-01 7,81E-02

2,1E-01 8,7E-02

2,2E-01 9,09E-02

2,2E-01

7,47E-02

2.2E-01 8,33E-02

2,3E-01 7,39E-02

2,3E-01 7,53E-02

2,4E-01 8,14E-02

2,4E-01 1,03E-01

2,4E-01 9,3E-02

2,4E-01 9,3E-02

2,4E-01 8,33E-02

2,5E-01 7,84E-02

2,5E-01 7,52E-02

2,5E-01 7,77E-02

2,6E-01

2,6E-01

0,2

2,6E-01 7,69E-02

2,6E-01 1,18E-01

2,6E-01 7,87E-02
SGMS2, SMPD3

2,12

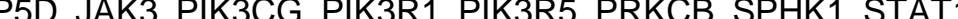

PIK3CG, PIK3R1, PIK3R5, PRKCB, PRKCQ, PTPN6, SOCS1

1,265 ADRB1, ATP2A2, ATP2A3, CAMK4, KNG1, PIK3CG, PIK3R1, PIK3R5, PRKCB, PRKCQ

1,387 ADCY7, BCAR1, CXCR4, FNBP1, PIK3CG, PIK3R1, PIK3R5 PLCB2, PRKCB, PRKCQ, PTK2, RHOD, $\mathrm{RHOH}$

CDC42, EREG, NCK2, PIK3CG, PIK3R1, PIK3R5, PRKCB, PRKCQ

-0,707 ABCG1, APOA1, IL18, IL18RAP, KNG1, MLXIPL, MMP9, SERPINA1, SREBF1, TLR3

2,449 PIK3CG, PIK3R1, PIK3R5, PRKCB, PRKCQ, STAT1

$-1,342$ CHEK2, E2F2, PPM1J, PPP2R2B, RAD1

ABCC3, ADCY7, IFNG, IKBKE, IL18, IL18RAP, IL4, IRAK3, PRKCB, PRKCQ, SLC10A2, SREBF1, TJP2

2,646 CASP8, FASLG, IKBKE, PIK3CG, PIK3R1, PIK3R5, TCF7

2,530 ADCY7, ATP2A2, ATP2A3, CAMK4, DRD4, KCNJ15, KCNJ5, PLCB2, PPM1J, PPP1CC, PPP2R2B, PRKCB, PRKCQ

BRCA2, CDKN2A, CGA, LEF1, MMP9, PIK3CG, PIK3R1, PIK3R5, WNT10A, WNT3, WNT3A

1,890 CDC42, IKBKE, PIK3CG, PIK3R1, PIK3R5, PRKCB, PRKCQ

IKBKE, TNFAIP3, TRAF1

$1,000 \quad$ CASP8, DHX58, IFIH1, IKBKE

-1,000 CDKN2A, PIK3CG, PIK3R1, PIK3R5

PLA2G4F, PLA2G7, PLAAT4, PLB1, PLCB2, PLD4

2,646 CAMK4, IKBKE, NFATC2, PIK3CG, PIK3R1, PIK3R5, PTK2B,

TRAF5

1,897 CDC42, FNBP1, IFNG, IL18, IL4, PIK3CG, PIK3R1, PIK3R5, RHOD

$\mathrm{RHOH}$

1,414 CDC42, ITGA4, NCK2, PIK3CG, PIK3R1, PIK3R5, PTK2, PTK2B

IL4

$\mathrm{FAAH}$

CDKN2A, E2F2, HDAC10, IKBKE, PIK3CG, PIK3R1, PIK3R5, TGFBR2

FMO3, UAP1

-0,378 BCL2L11, CASP8, CYCS, ENDOG, FASLG, IKBKE, PRKCQ 
Rac Signaling

4-1BB Signaling in T Lymphocytes

SAPK/JNK Signaling

eNOS Signaling

\section{FXR/RXR Activation}

\section{tRNA Splicing}

Signaling by Rho Family GTPases

Gai Signaling

Role of Tissue Factor in Cancer

ERK/MAPK Signaling

Alanine Degradation III

Alanine Biosynthesis II

Antioxidant Action of Vitamin C

VEGF Signaling

mTOR Signaling

Role of JAK2 in Hormone-like Cytokine Signaling NAD biosynthesis II (from tryptophan)

Telomerase Signaling

Cellular Effects of Sildenafil (Viagra)

Granzyme A Signaling

nNOS Signaling in Skeletal Muscle Cells

Cell Cycle Regulation by BTG Family Proteins

PI3K/AKT Signaling

Mitotic Roles of Polo-Like Kinase

\begin{tabular}{|c|c|}
\hline 2,7E-01 & 7,5E-02 \\
\hline 2,7E-01 & 9,68E-02 \\
\hline $\begin{array}{l}2,7 \mathrm{E}-01 \\
2,8 \mathrm{E}-01\end{array}$ & $\begin{array}{l}7,62 \mathrm{E}-02 \\
7,14 \mathrm{E}-02\end{array}$ \\
\hline 2,8E-01 & 7,3E-02 \\
\hline 2,8E-01 & 8,7E-02 \\
\hline 2,9E-01 & 6,83E-02 \\
\hline 2,9E-01 & 7,32E-02 \\
\hline $3,0 \mathrm{E}-01$ & 7,26E-02 \\
\hline $3,0 \mathrm{E}-01$ & 6,9E-02 \\
\hline $3,0 \mathrm{E}-01$ & 1,67E-01 \\
\hline $3,0 \mathrm{E}-01$ & 1,67E-01 \\
\hline $3,0 \mathrm{E}-01$ & 7,34E-02 \\
\hline 3,0E-01 & 7,34E-02 \\
\hline $3,1 \mathrm{E}-01$ & 6,83E-02 \\
\hline 3,2E-01 & 8,82E-02 \\
\hline 3,2E-01 & 8,82E-02 \\
\hline 3,2E-01 & 7,21E-02 \\
\hline 3,3E-01 & 6,99E-02 \\
\hline 3,3E-01 & 0,1 \\
\hline 3,3E-01 & 0,1 \\
\hline $3,4 \mathrm{E}-01$ & 8,57E-02 \\
\hline $3,4 \mathrm{E}-01$ & 6,98E-02 \\
\hline 3,4E-01 & 7,58E-02 \\
\hline
\end{tabular}

CDC42, ITGA4, NCF2, PIK3CG, PIK3R1, PIK3R5, PIP4K2A, PTK2, PTK2B

IKBKE, TNFRSF9, TRAF1

CDC42, LCK, MAP4K1, PIK3CG, PIK3R1, PIK3R5, RAC2, SH2D2A ADCY7, AQP7, CAMK4, CASP8, HSPA6, KNG1, LPAR2, PIK3CG, PIK3R1, PIK3R5, PRKCB, PRKCQ

APOA1, FBP1, IL18, KNG1, MLXIPL, PKLR, SDC1, SERPINA1, SLC10A2, SREBF1

PDE4A, PDE4D, PDE6A, PDE9A

ARHGEF16, CDC42, CDH16, CDH9, FNBP1, ITGA4, NCF2, PIK3CG, PIK3R1, PIK3R5, PIP4K2A, PTK2, PTK2B, RHOD, RHOH, WAS, WIPF1

ADCY7, ADORA3, CXCR2, DRD4, P2RY12, P2RY13, P2RY14, PTGER3, RGS10

BLK, CDC42, FGR, LCK, PDIA2, PIK3CG, PIK3R1, PIK3R5, PTK2B BCAR1, ITGA4, PIK3CG, PIK3R1, PIK3R5, PLA2G4F, PPM1J, PPP1CC, PPP2R2B, PRKCB, PTK2, PTK2B, RAC2, STAT1

GPT

GPT

CSF2RA, IKBKE, PLA2G4F, PLA2G7, PLAAT4, PLB1, PLCB2,

PLD4

PIK3CG, PIK3R1, PIK3R5, PRKCB, PTK2, PTK2B, PTPN6, $\mathrm{SH} 2 \mathrm{D} 2 \mathrm{~A}$

AKT1S1, DDIT4, FNBP1, PIK3CG, PIK3R1, PIK3R5, PLD4, PPM1J, PPP2R2B, PRKCB, PRKCQ, RHEB, RHOD, RHOH

PTPN6, SOCS1, STAT1

HAAO, IDO2, KMO

HDAC10, IL2RB, IL2RG, PIK3CG, PIK3R1, PIK3R5, PPM1J, PPP2R2B

ADCY7, CAMK4, KCNN2, KCNN4, MPRIP, MYH6, NPPA, PDE4A, PDE4D, PLCB2

GZMA, PRF1

CAMK4, RYR1

E2F2, PPM1J, PPP2R2B

IKBKE, INPP5D, ITGA4, JAK3, PIK3CG, PIK3R1, PPM1J, PPP2R2B, RHEB

CHEK2, KIF11, PLK2, PPM1J, PPP2R2B 
UVB-Induced MAPK Signaling

Spermine Biosynthesis

Choline Degradation I

Spermidine Biosynthesis I

Interferon Signaling

RhoGDI Signaling

Renal Cell Carcinoma Signaling

Type II Diabetes Mellitus Signaling

Epithelial Adherens Junction Signaling

14-3-3-mediated Signaling

D-myo-inositol $(1,4,5)$-Trisphosphate Biosynthesis

Huntington's Disease Signaling

p38 MAPK Signaling

Airway Pathology in Chronic Obstructive

Pulmonary Disease

Retinoate Biosynthesis II

Glycerol-3-phosphate Shuttle

Tight Junction Signaling

\section{FAK Signaling}

Sumoylation Pathway

Role of Cytokines in Mediating Communication

between Immune Cells

Phagosome Maturation

CCR3 Signaling in Eosinophils

Endothelin-1 Signaling

Inhibition of Angiogenesis by TSP1
3,4E-01 7,58E-02

3,4E-01 1,43E-01

3,4E-01 1,43E-01

3,4E-01 1,43E-01

3,5E-01 8,33E-02

3,5E-01 6,7E-02

3,6E-01 7,23E-02

3,6E-01 6,76E-02

3,6E-01 6,76E-02

3,7E-01 6,82E-02

3,7E-01 8,11E-02

3,7E-01 6,48E-02

3,8E-01 6,84E-02

3,8E-01 1,25E-01

3,8E-01 1,25E-01

3,8E-01 1,25E-01

3,9E-01 6,59E-02

3,9E-01 6,86E-02

3,9E-01 6,86E-02

3,9E-01 7,41E-02

3,9E-01 6,62E-02

3,9E-01 6,67E-02

4,0E-01 6,44E-02

4,0E-01 7,69E-02
PIK3CG, PIK3R1, PIK3R5, PRKCB, PRKCQ

AMD1

$\mathrm{CHDH}$

AMD1

IFNG, SOCS1, STAT1

0,000 ARHGAP4, ARHGAP9, ARHGDIB, ARHGEF16, CDC42, CDH16 CDH9, FNBP1, ITGA4, PIP4K2A, RHOD, RHOH

0,447 CDC42, MET, PIK3CG, PIK3R1, PIK3R5, UBD

2,449 IKBKE, PIK3CG, PIK3R1, PIK3R5, PKLR, PRKCB, PRKCQ, SLC27A2, SMPD3, SOCS1

CDC42, LEF1, MET, MYH6, NECTIN1, NOTCH2, TGFBR2, TUBA3C/TUBA3D, TUBB2A, WAS

1,890 AKT1S1, PIK3CG, PIK3R1, PIK3R5, PLCB2, PRKCB, PRKCQ, TUBA3C/TUBA3D, TUBB2A

PIP4K2A, PLCB2, PLD4

2,309 CACNA1B, CASP8, CYCS, GRM1, HDAC10, HSPA6, IGF1, PENK PIK3CG, PIK3R1, PIK3R5, PLCB2, PRKCB, PRKCQ, RPH3A, UBD FASLG, IL18, IL18RAP, IRAK3, MAP4K1, PLA2G4F, STAT1, TGFBR2 MMP9

RBP5

GPD1

CDC42, CLDN14, CLDN3, EPB41, F2RL2, MYH6, NECTIN1, PPM1J, PPP2R2B, TGFBR2, TJP2

BCAR1, ITGA4, PIK3CG, PIK3R1, PIK3R5, PTK2, WAS

0,000

ARHGDIB, FASLG, FNBP1, MYB, RHOD, RHOH, ZNF217

IFNG, IL18, IL23A, IL4

CTSK, CTSW, HLA-A, HLA-DRB1, HLA-DRB5, NCF2, PRDX6, RAB7B, TUBA3C/TUBA3D, TUBB2A

CAMK4, MPRIP, PIK3CG, PIK3R1, PIK3R5, PLA2G4F, PLCB2, PRKCB, PRKCQ

2,496 ADCY7, CASP8, PIK3CG, PIK3R1, PIK3R5, PLA2G4F, PLA2G7, PLAAT4, PLB1, PLCB2, PLD4, PRKCB, PRKCQ

MMP9, SDC1, TGFBR2 
Sertoli Cell-Sertoli Cell Junction Signaling

HIPPO signaling

IL-10 Signaling

Transcriptional Regulatory Network in Embryonic

Stem Cells

Inhibition of Matrix Metalloproteases

Leptin Signaling in Obesity

GABA Receptor Signaling

Melanoma Signaling

Role of JAK family kinases in IL-6-type Cytokine

Signaling

ErbB4 Signaling

RhoA Signaling

Docosahexaenoic Acid (DHA) Signaling

Glycolysis I

UVC-Induced MAPK Signaling

P2Y Purigenic Receptor Signaling Pathway

Embryonic Stem Cell Differentiation into Cardiac Lineages

Role of Wnt/GSK-3ß Signaling in the

Pathogenesis of Influenza

Ephrin Receptor Signaling

Neuregulin Signaling

Hereditary Breast Cancer Signaling

BMP signaling pathway

VEGF Family Ligand-Receptor Interactions

p53 Signaling

Relaxin Signaling

Role of IL-17F in Allergic Inflammatory Airway

Diseases

Cyclins and Cell Cycle Regulation

Heme Degradation
4,0E-01 6,45E-02

4,1E-01 6,82E-02

4,1E-01 6,94E-02

4,2E-01 7,5E-02

4,2E-01 7,5E-02

4,2E-01 6,74E-02

4,2E-01 6,85E-02

4,3E-01 7,02E-02

4,3E-01 $\quad 0,08$

4,4E-01 6,76E-02

4,4E-01 6,45E-02

4,4E-01 6,9E-02

4,5E-01 7,14E-02

4,5E-01 7,14E-02

4,5E-01 6,34E-02

1,667

4,5E-01 $\quad 0,1$

4,6E-01 6,58E-02

4,6E-01 6,21E-02

4,6E-01 6,45E-02

4,7E-01 6,25E-02

4,7E-01 6,49E-02

4,7E-01 6,38E-02

4,7E-01 6,31E-02

4,8E-01 6,17E-02

4,8E-01 6,82E-02

4,8E-01 6,41E-02

4,8E-01 9,09E-02

0,000
BCAR1, CDC42, CLDN14, CLDN3, EPB41, ITGA4, NECTIN1, RAB8B, TJP2, TUBA3C/TUBA3D, TUBB2A, WAS

$0,000 \quad P P M 1 J, P P P 1 C C$, PPP2R2B, STK4, TJP2, WWC1

CCR5, IKBKE, IL10RA, IL18, IL18RAP

EOMES, GATA4, HESX 1

MMP25, MMP9, SDC1

ADCY7, GHRL, PIK3CG, PIK3R1, PIK3R5, PLCB2

ADCY7, GABRR3, KCNN2, KCNN4, UBD

CDKN2A, PIK3CG, PIK3R1, PIK3R5

SOCS1, STAT1

2,236 PIK3CG, PIK3R1, PIK3R5, PRKCB, PRKCQ

0,707 ARHGAP4, ARHGAP9, IGF1, LPAR2, MPRIP, PIP4K2A, PTK2, PTK2B

CYCS, PIK3CG, PIK3R1, PIK3R5

ENO3, FBP1, PKLR

PRKCB, PRKCQ, SMPD3

ADCY7, P2RY12, P2RY2, PIK3CG, PIK3R1, PIK3R5, PLCB2, PRKCB, PRKCQ

GATA4

IFNG, LEF1, WNT10A, WNT3, WNT3A

1,265 ANGPT1, BCAR1, CDC42, CXCR4, ITGA4, NCK2, PIK3CG, PTK2 RAC2, WAS, WIPF1

0,447 EREG, ITGA4, MATK, PIK3R1, PRKCB, PRKCQ

BRCA2, CHEK2, FANCA, FANCD2, HDAC10, PIK3CG, PIK3R1, PIK3R5, UBD

$0,447 \quad$ BMP6, BMP7, CAMK4, FST, RUNX2

1,633 PIK3CG, PIK3R1, PIK3R5, PLA2G4F, PRKCB, PRKCQ

-1,134 CDKN2A, CHEK2, COQ8A, PIK3CG, PIK3R1, PIK3R5, TP53INP1

2,449 ADCY7, MMP9, PDE4A, PDE4D, PDE6A, PDE9A, PIK3CG, PIK3R1, PIK3R5, RLN2

CCL4, CXCL10, IGF1

HMOX2 
4-hydroxyproline Degradation I

IL-17A Signaling in Airway Cells

Gas Signaling

Role of NFAT in Cardiac Hypertrophy

Melanocyte Development and Pigmentation Signaling

Clathrin-mediated Endocytosis Signaling

CNTF Signaling

Gap Junction Signaling

PCP pathway

G Beta Gamma Signaling

Neurotrophin/TRK Signaling

Glutamate Receptor Signaling

Adipogenesis pathway

Non-Small Cell Lung Cancer Signaling

Endometrial Cancer Signaling

Assembly of RNA Polymerase III Complex

Glycerol Degradation

BER pathway

Glutathione Redox Reactions

Sonic Hedgehog Signaling

Tryptophan Degradation III (Eukaryotic)

Cell Cycle: G2/M DNA Damage Checkpoint Regulation

IL-17 Signaling

Ceramide Degradation

Glycoaminoglycan-protein Linkage Region Biosynthesis

Oncostatin M Signaling

Parkinson's Signaling

CMP-N-acetylneuraminate Biosynthesis I

(Eukaryotes)

Adenine and Adenosine Salvage III
4,8E-01

4,9E-01

4,9E-01

$5,0 \mathrm{E}-01$

$6,33 \mathrm{E}-02$

6,19E-02

6,03E-02

5,0E-01

6,19E-02

$5,0 \mathrm{E}-01$

0,06

5,1E-01

5,1E-01

$6,35 \mathrm{E}-02$

$6,01 \mathrm{E}-02$

5,1E-01

6,35E-02

$5,1 \mathrm{E}-01$

5,2E-01

$5,2 \mathrm{E}-01$

$6,12 \mathrm{E}-02$

$6,17 \mathrm{E}-02$

$6,25 E-02$

$5,4 \mathrm{E}-01 \quad 6,02 \mathrm{E}-02$

5,4E-01 6,06E-02

5,4E-01 7,69E-02

5,4E-01 7,69E-02

5,4E-01 7,69E-02

5,5E-01 6,45E-02

5,5E-01 6,45E-02

5,5E-01 6,12E-02

5,5E-01 6,12E-02

5,6E-01 5,88E-02

5,7E-01 7,14E-02

5,7E-01 7,14E-02

6,0E-01 5,88E-02

6,2E-01 6,25E-02

6,4E-01 5,88E-02

6,4E-01 5,88E-02
HOGA1

IKBKE, JAK3, PIK3CG, PIK3R1, PIK3R5

$-0,378$ ADCY7, ADRB1, HRH2, PTGDR, PTH1R, RYR1, VIPR2

ADCY7, CAMK4, GATA4, HDAC10, IGF1, PIK3CG, PIK3R1, PIK3R5, PLCB2, PRKCB, PRKCQ, TGFBR2

1,342 ADCY7, PIK3CG, PIK3R1, PIK3R5, PTPN6, SOX10

APOA1, CDC42, F2R, IGF1, ITGB2, ITGB7, MET, PIK3CG, PIK3R1, PIK3R5, SERPINA1, UBD

2,000 PIK3CG, PIK3R1, PIK3R5, STAT1

ADCY7, ADRB1, PIK3CG, PIK3R1, PIK3R5, PLCB2, PRKCB,

PRKCQ, TJP2, TUBA3C/TUBA3D, TUBB2A

SDC1, WNT10A, WNT3, WNT3A

0,816 BTK, CDC42, KCNJ5, PIK3CG, PRKCB, PRKCQ

1,000 CDC42, PIK3CG, PIK3R1, PIK3R5, SORCS1

CAMK4, GRIK2, GRIK3, GRM1

BMP7, CDKN2A, DGKD, ERCC2, HDAC10, SREBF1, TCF7, TXNIP

1,000

CDKN2A, PIK3CG, PIK3R1, PIK3R5, STK4

LEF1, PIK3CG, PIK3R1, PIK3R5

GTF3A

GPD1

LIG1

GPX3, PRDX6

GLIS1, GLIS2

HAAO, IDO2, KMO

AURKA, CDKN2A, CHEK2

CXCL10, CXCL11, PIK3CG, PIK3R1, PIK3R5

NAAA

B3GAT1

JAK3, STAT1

CYCS

NANP

ADAT3 
Pregnenolone Biosynthesis

Circadian Rhythm Signaling

Synaptic Long Term Potentiation

Amyotrophic Lateral Sclerosis Signaling

NRF2-mediated Oxidative Stress Response

PPARa/RXRa Activation

Aryl Hydrocarbon Receptor Signaling

LPS/IL-1 Mediated Inhibition of RXR Function

Mitochondrial Dysfunction

PXR/RXR Activation

TR/RXR Activation

RAR Activation

a-Adrenergic Signaling

MIF Regulation of Innate Immunity

IL-22 Signaling

Mechanisms of Viral Exit from Host Cells

HIF1a Signaling

Melatonin Signaling

Cardiac Hypertrophy Signaling

CDK5 Signaling

Corticotropin Releasing Hormone Signaling

Maturity Onset Diabetes of Young (MODY)

Signaling

GNRH Signaling

ATM Signaling

Antiproliferative Role of Somatostatin Receptor 2

Androgen Signaling

Aldosterone Signaling in Epithelial Cells

CREB Signaling in Neurons

Prostate Cancer Signaling
6,4E-01

$1,0 \mathrm{E}+00$

$1,0 \mathrm{E}+00$

$1,0 \mathrm{E}+00$

$1,0 \mathrm{E}+00$

$1,0 \mathrm{E}+00$

1,0E+00 3,42E-02

$1,0 \mathrm{E}+00 \quad 3,96 \mathrm{E}-02$

1,0E+00 3,19E-02

$1,0 \mathrm{E}+00 \quad 1,43 \mathrm{E}-02$

$1,0 \mathrm{E}+00 \quad 5,71 \mathrm{E}-02$

$1,0 \mathrm{E}+00 \quad 3,55 \mathrm{E}-02$

$1,0 \mathrm{E}+00 \quad 5,15 \mathrm{E}-02$

$1,0 \mathrm{E}+00 \quad 4,55 \mathrm{E}-02$

$1,0 \mathrm{E}+00 \quad 4,17 \mathrm{E}-02$

1,0E+00 4,88E-02

$1,0 \mathrm{E}+00 \quad 5,13 \mathrm{E}-02$

$1,0 \mathrm{E}+00 \quad 5,19 \mathrm{E}-02$

1,0E+00 5,33E-02

$1,0 \mathrm{E}+00 \quad 3,88 \mathrm{E}-02$

$1,0 \mathrm{E}+00 \quad 4,13 \mathrm{E}-02$

$1,0 \mathrm{E}+00$

0,04

1,0E+00 4,44E-02

$1,0 \mathrm{E}+00 \quad 3,75 \mathrm{E}-02$

$1,0 \mathrm{E}+00 \quad 5,06 \mathrm{E}-02$

$1,0 \mathrm{E}+00 \quad 4,39 \mathrm{E}-02$

$1,0 \mathrm{E}+00 \quad 5,11 \mathrm{E}-02$

1,0E+00 5,73E-02

$1,0 \mathrm{E}+00$
CAMK4, GRM1, PLCB2, PPP1CC, PPP1R1A, PRKCB, PRKCQ

CYCS, GRIK2, GRIK3, IGF1, PIK3CG, PIK3R1, PIK3R5

DNAJB5, FOSL1, NQO2, PIK3CG, PIK3R1, PIK3R5, PRKCB PRKCQ

0,000 ADCY7, APOA1, GPD1, IKBKE, IL18RAP, PLCB2, PRKCB TGFBR2

CDKN2A, CHEK2, FASLG, NQO2, TFF1

ABCC3, ABCG1, CHST1, CHST15, FMO3, IL18, IL18RAP SLC27A2, SREBF1

ATP5MC1, CASP8, COX5A, CYCS, FURIN, LRRK2

ABCC3

ADRB1, CAMK4, PIK3CG, PIK3R1, PIK3R5, SREBF1

ADCY7, ERCC2, PIK3CG, PIK3R1, PRKCB, PRKCQ, RBP5

1,342 ADCY7, CAMK4, PRKCB, PRKCQ, PYGM

CD74, PLA2G4F

STAT1

PRKCB, PRKCQ

EGLN1, MMP25, MMP9, PIK3CG, PIK3R1, PIK3R5

2,000 CAMK4, PLCB2, PRKCB, PRKCQ

1,941 ADCY7, ADRB1, CAMK4, FNBP1, GATA4, IGF1, PIK3CG, PIK3R1, PIK3R5, PLCB2, RHOD, RHOH, TGFBR2

1,000 ADCY7, PPM1J, PPP1CC, PPP2R2B

2,000 ADCY7, CAMK4, FASLG, PRKCB, PRKCQ

PKLR

0,816

ADCY7, CDC42, PLCB2, PRKCB, PRKCQ, PTK2

CHEK2, FANCD2, PPP2R2B

2,000 PIK3CG, PIK3R1, PIK3R5, PTPN6

$1,000 \quad$ CAMK4, ERCC2, PRKCB, PRKCQ, SHBG

2,646 DNAJB5, HSPA6, PIK3CG, PIK3R1, PIK3R5, PIP4K2A, PLCB2,

PRKCB, PRKCQ

2,111 ADCY7, CAMK4, GRIK2, GRIK3, GRM1, PIK3CG, PIK3R1, PIK3R5, PLCB2, PRKCB, PRKCQ

LEF1, PIK3CG, PIK3R1, PIK3R5 
Thyroid Cancer Signaling

Bladder Cancer Signaling

ERK5 Signaling

G Protein Signaling Mediated by Tubby

EIF2 Signaling

AMPK Signaling

Regulation of elF4 and p70S6K Signaling

Estrogen-Dependent Breast Cancer Signaling

IL-1 Signaling

Role of Lipids/Lipid Rafts in the Pathogenesis of nfluenza

Antiproliferative Role of TOB in T Cell Signaling

Extrinsic Prothrombin Activation Pathway

MIF-mediated Glucocorticoid Regulation

Cell Cycle Control of Chromosomal Replication

Assembly of RNA Polymerase II Complex

Role of IL-17A in Arthritis

L-17A Signaling in Fibroblasts

NGF Signaling

Mouse Embryonic Stem Cell Pluripotency

nNOS Signaling in Neurons

ErbB2-ErbB3 Signaling

Estrogen-mediated S-phase Entry

Heparan Sulfate Biosynthesis

Fatty Acid Activation

Sphingosine and Sphingosine-1-phosphate

Metabolis

Retinoate Biosynthesis

Thyroid Hormone Metabolism II (via Conjugation and/or Degradation)

Nicotine Degradation III

Heparan Sulfate Biosynthesis (Late Stages)

Ubiquinol-10 Biosynthesis (Eukaryotic)

CDP-diacylglycerol Biosynthesis I

The Visual Cycle
$1,0 \mathrm{E}+00$

$1,0 \mathrm{E}+00$

2,44E-02

$3,45 \mathrm{E}-02$

$1,0 \mathrm{E}+00 \quad 3,12 \mathrm{E}-02$

$1,0 \mathrm{E}+00 \quad 5,71 \mathrm{E}-02$

$1,0 \mathrm{E}+00 \quad 2,68 \mathrm{E}-02$

$1,0 \mathrm{E}+00 \quad 3,4 \mathrm{E}-02$

$1,0 \mathrm{E}+00 \quad 4,35 \mathrm{E}-02$

$1,0 \mathrm{E}+00 \quad 4,94 \mathrm{E}-02$

$1,0 \mathrm{E}+00 \quad 3,19 \mathrm{E}-02$

$1,0 \mathrm{E}+00 \quad 4,35 \mathrm{E}-02$

1,0E $+00 \quad 3,85 \mathrm{E}-02$

$1,0 \mathrm{E}+00 \quad 5,56 \mathrm{E}-02$

$1,0 \mathrm{E}+00 \quad 5,71 \mathrm{E}-02$

$1,0 \mathrm{E}+00 \quad 5,26 \mathrm{E}-02$

$1,0 \mathrm{E}+00 \quad 0,02$

1,0E+00 5,8E-02

$1,0 \mathrm{E}+00 \quad 2,86 \mathrm{E}-02$

$1,0 \mathrm{E}+00 \quad 4,88 \mathrm{E}-02$

$1,0 \mathrm{E}+00 \quad 5,66 \mathrm{E}-02$

$1,0 \mathrm{E}+00 \quad 5,77 \mathrm{E}-02$

$1,0 \mathrm{E}+00 \quad 5,8 \mathrm{E}-02$

1,0E+00 4,17E-02

$1,0 \mathrm{E}+00 \quad 4,17 \mathrm{E}-02$

$1,0 \mathrm{E}+00 \quad 5,26 \mathrm{E}-02$

$1,0 \mathrm{E}+00 \quad 5,26 \mathrm{E}-02$

$1,0 \mathrm{E}+00 \quad 2,63 \mathrm{E}-02$

$1,0 \mathrm{E}+00 \quad 3,28 \mathrm{E}-02$

$1,0 \mathrm{E}+00 \quad 3,39 \mathrm{E}-02$

1,0E+00 3,53E-02

$1,0 \mathrm{E}+00 \quad 3,12 \mathrm{E}-02$

1,0E+00 2,86E-02

$1,0 \mathrm{E}+00 \quad 3,12 \mathrm{E}-02$

\section{LEF1}

CDKN2A, MMP25, MMP9

FOSL1, SH2D2A

LCK, PLCB2

0,816

0,378

1,633

PIK3CG, PIK3R1, PIK3R5, PPP1CC, RPL27A, SREBF1

ADRB1, AKT1S1, PIK3CG, PIK3R1, PIK3R5, PPM1J, PPP2R2B

EIF4EBP2, ITGA4, PIK3CG, PIK3R1, PIK3R5, PPM1J, PPP2R2B

IGF1, PIK3CG, PIK3R1, PIK3R5

ADCY7, IKBKE, IRAK3

IFNG

TGFBR2

F13A1

CD74, PLA2G4F

CHEK2, LIG1

ERCC2

CCL5, PIK3CG, PIK3R1, PIK3R5

IKBKE

1,633 CDC42, IKBKE, PIK3CG, PIK3R1, PIK3R5, SMPD3

1,633 JAK3, LEF1, PIK3CG, PIK3R1, PIK3R5, WNT3A

CAMK4, PRKCB, PRKCQ

2,000 JAK3, PIK3CG, PIK3R1, PIK3R5

E2F2

B3GAT1, CHST1, CHST15, PRDX6

SLC27A2

NAAA

RBP5

LARGE1, LARGE2

LARGE1, LARGE2

CHST1, CHST15, PRDX6

MICAL1

LPCAT3

RBP5 
Melatonin Degradation I

Y-linolenate Biosynthesis II (Animals)

NAD Salvage Pathway II

Pyridoxal 5'-phosphate Salvage Pathway

Pyrimidine Deoxyribonucleotides De Novo

Biosynthesis I

Triacylglycerol Degradation

Choline Biosynthesis III

Chondroitin Sulfate Biosynthesis

Dermatan Sulfate Biosynthesis

Glycogen Degradation III

Glutathione-mediated Detoxification

1D-myo-inositol Hexakisphosphate Biosynthesis I

(Mammalian)

NAD Phosphorylation and Dephosphorylation

Dermatan Sulfate Biosynthesis (Late Stages)

Phosphatidylglycerol Biosynthesis II (Non-

plastidic)

Glycogen Degradation II

Nicotine Degradation II

Superpathway of D-myo-inositol $(1,4,5)$ -

trisphosphate Metabolism

Purine Nucleotides Degradation II (Aerobic)

Mitochondrial L-carnitine Shuttle Pathway

D-myo-inositol $(1,3,4)$-trisphosphate Biosynthesis

Serotonin Degradation

Phenylalanine Degradation IV (Mammalian, via

Side Chain)

D-myo-inositol $(1,4,5)$-trisphosphate Degradation

Retinol Biosynthesis

Purine Ribonucleosides Degradation to Ribose-1phosphate

Triacylglycerol Biosynthesis

Salvage Pathways of Pyrimidine Ribonucleotides

Gluconeogenesis I

$\begin{array}{cc}1,0 \mathrm{E}+00 & 2,74 \mathrm{E}-02 \\ 1,0 \mathrm{E}+00 & 4,17 \mathrm{E}-02 \\ 1,0 \mathrm{E}+00 & 2,86 \mathrm{E}-02 \\ 1,0 \mathrm{E}+00 & 2,67 \mathrm{E}-02 \\ 1,0 \mathrm{E}+00 & 2,44 \mathrm{E}-02 \\ 1,0 \mathrm{E}+00 & 5,08 \mathrm{E}-02 \\ 1,0 \mathrm{E}+00 & 4,55 \mathrm{E}-02 \\ 1,0 \mathrm{E}+00 & 4,05 \mathrm{E}-02 \\ 1,0 \mathrm{E}+00 & 0,04 \\ 1,0 \mathrm{E}+00 & 0,05 \\ 1,0 \mathrm{E}+00 & 2,44 \mathrm{E}-02 \\ 1,0 \mathrm{E}+00 & 3,7 \mathrm{E}-02 \\ 1,0 \mathrm{E}+00 & 0,05 \\ 1,0 \mathrm{E}+00 & 3,85 \mathrm{E}-02 \\ 1,0 \mathrm{E}+00 & 2,44 \mathrm{E}-02 \\ 1,0 \mathrm{E}+00 & 5,56 \mathrm{E}-02 \\ 1,0 \mathrm{E}+00 & 4,11 \mathrm{E}-02 \\ 1,0 \mathrm{E}+00 & 3,12 \mathrm{E}-02 \\ 1,0 \mathrm{E}+00 & 2,7 \mathrm{E}-02 \\ 1,0 \mathrm{E}+00 & 4,76 \mathrm{E}-02 \\ 1,0 \mathrm{E}+00 & 0,04 \\ 1,0 \mathrm{E}+00 & 2,2 \mathrm{E}-02 \\ 1,0 \mathrm{E}+00 & 3,12 \mathrm{E}-02 \\ 1,0 \mathrm{E}+00 & 4,55 \mathrm{E}-02 \\ 1,0 \mathrm{E}+00 & 4,08 \mathrm{E}-02 \\ 1,0 \mathrm{E}+00 & 0,05 \\ 1,0 \mathrm{E}+00 & 1,72 \mathrm{E}-02 \\ 1,0 \mathrm{E}+00 & 4,46 \mathrm{E}-02 \\ 1,0 \mathrm{E}+00 & 4,26 \mathrm{E}-02 \\ & \\ 1,00 & \\ 1,00 & \end{array}$

\author{
LARGE1, LARGE2 \\ SLC27A2 \\ ACP5 \\ PIM1, PRKCQ \\ APOBEC3G
}

CEL, FAAH, PRDX6

PLD4

B3GAT1, CHST1, CHST15

B3GAT1, CHST1, CHST15

PYGM

ANPEP

INPP5D

ACP5

CHST1, CHST15

LPCAT3

PYGM

FMO3, LARGE1, LARGE2

INPP5D

ADAT3

SLC27A2

INPP5D

LARGE1, LARGE2

SLC27A2

INPP5D

CEL, RBP5

ADAT3

LPCAT3

APOBEC2, APOBEC3G, APOBEC3H, PIM1, PRKCQ

ENO3, FBP1 
Histamine Degradation

Chondroitin Sulfate Biosynthesis (Late Stages)

Salvage Pathways of Pyrimidine

Deoxyribonucleotides

Adenosine Nucleotides Degradation II

Superpathway of Melatonin Degradation

Histidine Degradation VI

Stearate Biosynthesis I (Animals)

UDP-N-acetyl-D-galactosamine Biosynthesis II

Fatty Acid $\beta$-oxidation I

DNA damage-induced 14-3-3 $\sigma$ Signaling

Regulation of Cellular Mechanics by Calpain

Protease

Remodeling of Epithelial Adherens Junctions

STAT3 Pathway

Oxidative Phosphorylation

Wnt/Ca+ pathway

Estrogen Receptor Signaling

EGF Signaling

Cell Cycle: G1/S Checkpoint Regulation

Nucleotide Excision Repair Pathway

Protein Ubiquitination Pathway

FGF Signaling

Endoplasmic Reticulum Stress Pathway

Xenobiotic Metabolism Signaling

Serotonin Receptor Signaling

Insulin Receptor Signaling

Phototransduction Pathway

PPAR Signaling

IGF-1 Signaling

Dopamine Receptor Signaling

Notch Signaling

Hypoxia Signaling in the Cardiovascular System

$\begin{array}{cc}1,0 \mathrm{E}+00 & 3,03 \mathrm{E}-02 \\ 1,0 \mathrm{E}+00 & 3,39 \mathrm{E}-02 \\ 1,0 \mathrm{E}+00 & 0,05 \\ 1,0 \mathrm{E}+00 & 3,57 \mathrm{E}-02 \\ 1,0 \mathrm{E}+00 & 2,3 \mathrm{E}-02 \\ 1,0 \mathrm{E}+00 & 4,55 \mathrm{E}-02 \\ 1,0 \mathrm{E}+00 & 1,82 \mathrm{E}-02 \\ 1,0 \mathrm{E}+00 & 3,85 \mathrm{E}-02 \\ 1,0 \mathrm{E}+00 & 2,22 \mathrm{E}-02 \\ 1,0 \mathrm{E}+00 & 5,26 \mathrm{E}-02 \\ 1,0 \mathrm{E}+00 & 3,45 \mathrm{E}-02 \\ 1,0 \mathrm{E}+00 & 4,35 \mathrm{E}-02 \\ 1,0 \mathrm{E}+00 & 5,41 \mathrm{E}-02 \\ 1,0 \mathrm{E}+00 & 2,52 \mathrm{E}-02 \\ 1,0 \mathrm{E}+00 & 3,33 \mathrm{E}-02 \\ 1,0 \mathrm{E}+00 & 1,56 \mathrm{E}-02 \\ 1,0 \mathrm{E}+00 & 5,56 \mathrm{E}-02 \\ 1,0 \mathrm{E}+00 & 4,69 \mathrm{E}-02 \\ 1,0 \mathrm{E}+00 & 2,86 \mathrm{E}-02 \\ 1,0 \mathrm{E}+00 & 3,86 \mathrm{E}-02 \\ 1,0 \mathrm{E}+00 & 5,32 \mathrm{E}-02 \\ 1,0 \mathrm{E}+00 & 4,76 \mathrm{E}-02 \\ 1,0 \mathrm{E}+00 & 4,48 \mathrm{E}-02 \\ 1,0 \mathrm{E}+00 & 1,89 \mathrm{E}-02 \\ 1,0 \mathrm{E}+00 & 3,4 \mathrm{E}-02 \\ 1,0 \mathrm{E}+00 & 4,92 \mathrm{E}-02 \\ 1,0 \mathrm{E}+00 & 3,23 \mathrm{E}-02 \\ 1,0 \mathrm{E}+00 & 5,56 \mathrm{E}-02 \\ 1,0 \mathrm{E}+00 & 5,49 \mathrm{E}-02 \\ 1,0 \mathrm{E}+00 & 5,26 \mathrm{E}-02 \\ 1,0 \mathrm{E}+00 & 1,54 \mathrm{E}-02 \\ & \end{array}$

HNMT

CHST1, CHST15

APOBEC3G

ADAT3

LARGE1, LARGE2

MICAL1

SLC27A2

UAP

SLC27A2

RAD1

ITGA4, PTK2

MET, TUBA3C/TUBA3D, TUBB2A

-1,000 PIM1, PTPN6, SOCS1, TGFBR2

ATP5MC1, COX5A, CYCS

NFATC2, PLCB2

ERCC2, RUNX2

2,000 PIK3CG, PIK3R1, PIK3R5, STAT1

CDKN2A, E2F2, HDAC10

$\mathrm{ERCC2}$

BAG1, DNAJB5, HLA-A, HSPA6, IFNG, UBD, UBE2L6, USP2, USP31, USP51

0,447 MET, PIK3CG, PIK3R1, PIK3R5, PTPN6

ERN1

ABCC3, CAMK4, CHST1, CHST15, FMO3, NQO2, PIK3CG, PIK3R1, PIK3R5, PPM1J, PPP2R2B, PRKCB, PRKCQ

ADCY7

0,447 INPP5D, PIK3CG, PIK3R1, PIK3R5, PPP1CC

PDE6A, RGS9, RGS9BP

IKBKE, IL18, IL18RAP

0,816 IGF1, PIK3CG, PIK3R1, PIK3R5, PTK2, SOCS1

ADCY7, DRD4, PPM1J, PPP1CC, PPP2R2B

FURIN, NOTCH2

UBE2L6 


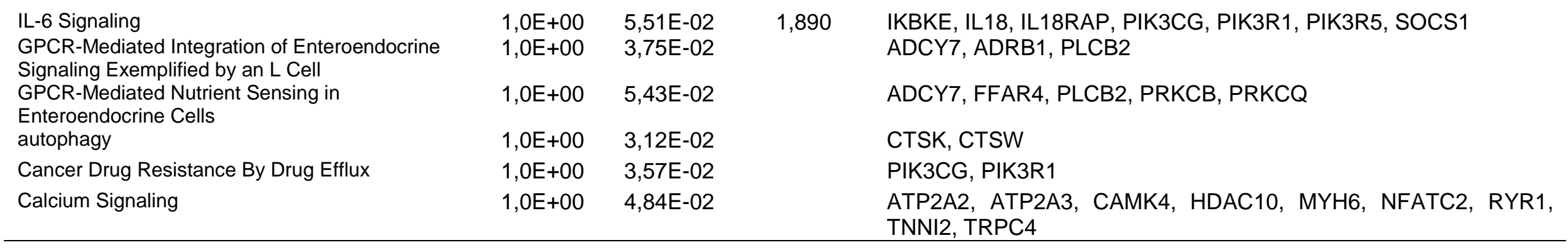

P-value of the overlap between DEGs and canonical pathway gene lists was calculated with Fisher's exact test. Ratio is the percentage of DEGs in each canonical pathway 